\title{
Adipogenic function of tetranectin mediated by enhancing mitotic clonal expansion via ERK signaling
}

\author{
Seulgi Go ${ }^{1, \#}$, Jihyun Park ${ }^{1, \#}$, Safikur Rahman ${ }^{2}$, Juno Jin ${ }^{1}$, Inho Choi ${ }^{1} \mathcal{E}$ Jihoe Kim ${ }^{1, *}$ \\ ${ }^{1}$ Department of Medical Biotechnology and Research Institute of Cell Culture, Yeungnam University, Gyeongsan 38541, Korea, \\ ${ }^{2}$ Department of Botany, Munshi Singh College, BR Ambedkar Bihar University, Muzaffarpur, Bihar 845401, India
}

\begin{abstract}
Tetranectin (TN), an adipogenic serum protein, enhances adipocyte differentiation, however, its functional mechanism has yet to be elucidated. In the present study, we investigated the adipogenic function of TN by using medium containing TN-depleted fetal bovine serum (TN-del-FBS) and recombinant mouse TN (mTN). The adipocyte differentiation of 3T3-L1 cells was significantly enhanced by mTN supplementation essentially at differentiation induction, which indicated a potential role of the protein in the early differentiation phase. The adipogenic effect of mTN was more significant with insulin in the differentiation induction cocktail, implicating their close functional relationship. mTN enhanced not only the proliferation of growing cells, but also mitotic clonal expansion (MCE) that is a prerequisite for adipocyte differentiation in the early phase. Consistently, mTN increased the phosphorylation of ERK in the early phase of adipocyte differentiation. Results of this study demonstrate that the adipogenic function of $\mathrm{mTN}$ is mediated by enhancing MCE via ERK signaling. [BMB Reports 2021; 54(7): 374-379]
\end{abstract}

\section{INTRODUCTION}

Tetranectin (TN) was discovered in human serum as a plasminogen (Plg)-binding protein (1). TN binds to the kringle-4 domain of Plg and enhances the Plg-activator-catalyzed activation of Plg into plasmin (Pln) (2). Although the physiological role of $\mathrm{TN}$ is not yet clearly understood, $\mathrm{TN}$ has been suggested as a potential serum marker of various cancers (3-6). Serum TN levels are estimated to be $\sim 10 \mathrm{mg} / \mathrm{L}$ in healthy individuals, but are significantly lower in cancer patients. Moreover, the protein was accumulated in the extracellular

*Corresponding author. Tel: +82-53-810-3032; Fax: +82-53-810-4769; E-mail: kimjihoe@ynu.ac.kr

\#These authors contributed equally to this work.

https://doi.org/10.5483/BMBRep.2021.54.7.024

Received 16 February 2021, Revised 3 March 2021, Accepted 6 March 2021

Keywords: Adipocyte differentiation, ERK, Mitotic clonal expansion, Serum protein, Tetranectin matrix (ECM) of various carcinomas, which suggested that the protein could be involved in ECM remodeling during cancer development and progression (7-9). Apart from its relationship with cancers, TN is believed to be involved in bone and muscle development, as it shows high expression levels in osteogenesis and myogenesis $(10,11)$. In addition, TN was also identified to be involved in the regulation of adipocyte differentiation (12).

Mouse-derived 3T3-L1 preadipocytes have been extensively used as a model cell line in studies of adipocyte differentiation, which have revealed many underlying mechanisms (13). The adipocyte differentiation of 3T3-L1 cells requires post-confluent and growth-arrested cells, and the following induction of differentiation in media containing fetal bovine serum (FBS). Differentiation induction is accomplished by the addition of a cocktail comprising 3-isobutyl-1-methylxanthine (MIX), dexamethasone (DEX), and insulin. Upon the induction, growth-arrested cells undergo the essential process mitotic clonal expansion (MCE) in the early differentiation phase (14). Then another growth arrest and terminal differentiation proceeds with lipid accumulation. MCE is regulated via the MEK/ERK signaling pathway, which includes the activation of ERK by phosphorylation upon differentiation induction $(15,16)$. MIX, a component of the induction cocktail, was determined to be a principle activator of ERK phosphorylation (17). Furthermore, as another activator, insulin further increases the phosphorylation of ERK during the early phase of adipocyte differentiation.

Previously, we identified TN as an adipogenic protein in FBS that enhances the adipocyte differentiation of 3T3-L1 cells (12, 18). In another study, the conserved kringle-4-binding domain of mouse TN (mTN) was determined to be essential for its adipogenic function (19). However, adipocyte differentiation was not affected by Plg, Pln, or Plg-activator, indicating that the adipogenic function of $\mathrm{mTN}$ is not mediated by the activation of Plg. In this study, to elucidate the mechanism of its adipogenic function, we used the recombinant mouse protein mTN, and medium containing TN-depleted FBS (TNdel-FBS medium). Significant adipogenic effect was obtained by mTN supplementation essentially at the differentiation induction, which indicated that the protein plays a role in the early phase of adipocyte differentiation. Moreover, we found that mTN enhanced MCE and increased the phosphorylation of ERK in 
the early phase of adipocyte differentiation. Results in this study demonstrate that the adipogenic function of $\mathrm{mTN}$ is mediated by enhancing MCE via ERK signaling.

\section{RESULTS}

\section{Adipogenic effect of mTN in the early phase of adipocyte differentiation}

The adipogenic effect of mTN was examined by inducing adipocyte differentiation of post-confluent and growth-arrested 3T3-L1 cells as described in the scheme (Fig. 1A). Adipocyte differentiation in TN-del-FBS medium was significantly decreased to $<50 \%$ of the control level obtained in the FBS medium following the standard differentiation process (Fig. 1B). Supplementation of TN-del-FBS medium with mTN markedly enhanced adipocyte differentiation, consistently indicating the adipogenic effect of the protein (Fig. 1B) (19). mTN supplementation on the day 0 with the induction cocktail MDI (E1) resulted in $180 \%$ adipocyte differentiation compared to the control level obtained in TN-del-FBS medium without protein supplementation. mTN supplementation from the day 0 to the day 5 (E2) and the day 0 to the day 8 (E3) resulted in similar adipogenic effect (170-180\% differentiation). After two days post-induction, however, mTN did not show any significant adipogenic effect (Fig. 1B, E4-E6). In FBS medium, mTN supplementation did not change the adipocyte differentiation, likely due to the presence of sufficient amount of TN derived from FBS (see the discussion).

Enhancement of adipocyte differentiation by insulin and mTN The effects of MIX, DEX, and insulin as components of the induction cocktail were examined on adipocyte differentiation in TN-del-FBS medium. Any significant adipocyte differentiation was not observed by induction with MIX, DEX, or insulin alone or with mTN supplementation (data not shown). Both MIX and DEX (MD) were essential for inducing adipocyte differentiation at a detectable level (Fig. 2A). Insulin with MD in the induction cocktail significantly increased adipocyte differentiation with a dose-dependency (Fig. 2A and B). The adipogenic effect of mTN was observed by induction with $M D$, although the effect was lower than that observed by induction with MDI (Fig. 2A). Moreover, the adipogenic effect of mTN appeared to be augmented by increasing the concentration of insulin in the induction cocktail (Fig. 2B). The expression of adipogenic marker genes was significantly increased by mTN supplementation, which confirmed the adipogenic effect of the protein (Fig. 2C and D).

\section{mTN enhances cell proliferation and MCE}

The effect of mTN on cell proliferation was examined by growing 3T3-L1 cells in TN-del-FBS medium. mTN dosedependently enhanced the proliferation of 3T3-L1 cells, which showed $\sim 50 \%$ increase in cell numbers at $7 \mu \mathrm{M}$ protein concentration (Fig. 3A). Next, the effect of mTN on MCE was examined by determining the cell numbers after inducing adipocyte differentiation of growth-arrested 3T3-L1 cells in $\mathrm{TN}$-del-FBS medium. The cell numbers were increased 1.3and 1.5-fold at $24 \mathrm{~h}$ and $48 \mathrm{~h}$, respectively, by induction with MDI, indicating MCE (Fig. 3B and C). In addition, mTN further increased the cell number by 1.7 -fold at $48 \mathrm{~h}$, but the change was insignificant at $24 \mathrm{~h}$. Differentiation induction with MD caused insignificant changes in cell numbers with slight decreases, however, mTN supplementation significantly increased the cell number at $48 \mathrm{~h}$ (Fig. 3C).

\section{mTN increases the phosphorylation of ERK}

ERK phosphorylation was analyzed for $48 \mathrm{~h}$ after the induction of adipocyte differentiation of 3T2-L1 cells in TN-del-FBS medium. The analysis showed a rapid increase in ERK expression and phosphorylation upon inducing differentiation with MDI (Fig. 4A). Phosphorylation of ERK increased, reaching a maximum level at $30 \mathrm{~min}$ and subsequently decreasing to a basal level at $6 \mathrm{~h}$, while the change in ERK expression was insignificant (Fig. $4 \mathrm{~A}$ and $\mathrm{B})$. The second maximum level of ERK phosphoryla-
A


B B
Fig. 1. Adipogenic effect of mTN in the early phase of adipocyte differentiation. (A) Scheme for the adipocyte differentiation of 3T3-L1 cells following the standard process (upper). TN-delFBS media were supplemented with 5 $\mu \mathrm{M}$ mTN on different days as indicated for different days (E1-E6). (B) Oil red $\mathrm{O}$ staining of differentiated cells (upper) and determination of lipid contents by isopropanol extraction (lower). E1-E6 are the same as in the scheme A. Data with $\mathrm{P}$ values $<0.05(*)$ are indicated. 


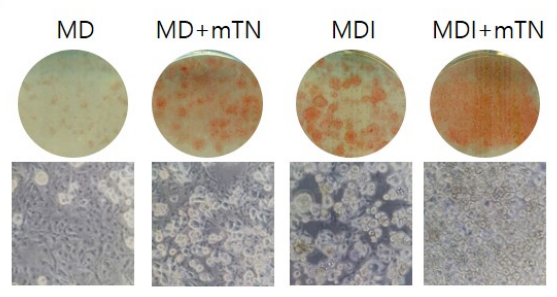

C

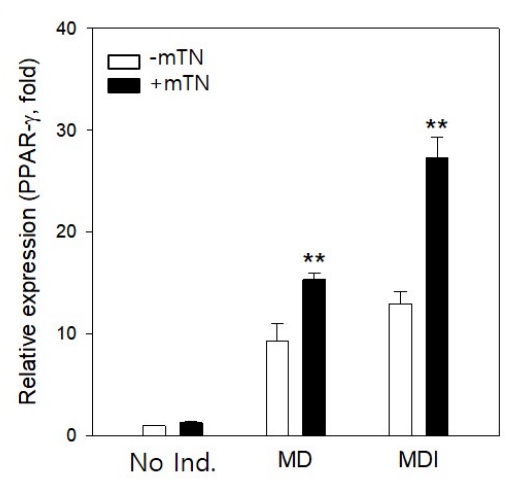

A

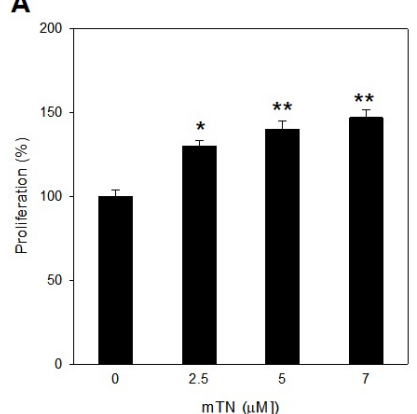



D



Fig. 2. Enhancement of adipocyte differentiation by insulin and mTN. (A) Adipocyte differentiation of 3T3-L1 cells induced with $5 \mu \mathrm{M} \mathrm{mTN}$ and the indicated induction cocktails: MD, 3-isobutyl1-methylxanthine + dexamethasone; $\mathrm{MDI}$, $\mathrm{MD}+5 \mu \mathrm{g} / \mathrm{ml}$ insulin. Adipocyte differentiation was estimated by oil red $\mathrm{O}$ staining (upper) and microscopy (lower). (B) Induction of differentiation with $\mathrm{MD}$ and the indicated concentrations of insulin $\pm 5 \mu \mathrm{M} \mathrm{mTN}$. (C and D) Determination of adipogenic marker gene expression by quantitative real-time PCR. No ind., no induction. Data with $\mathrm{P}$ values $<0.05$ $(*)$ and $<0.01(* *)$ are indicated.
B

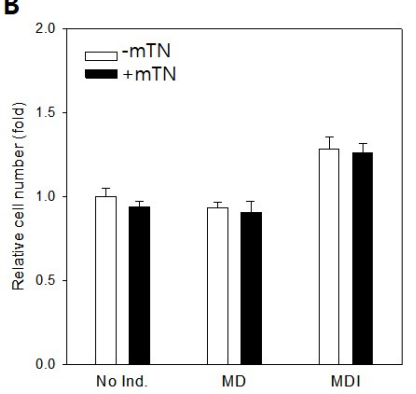

C

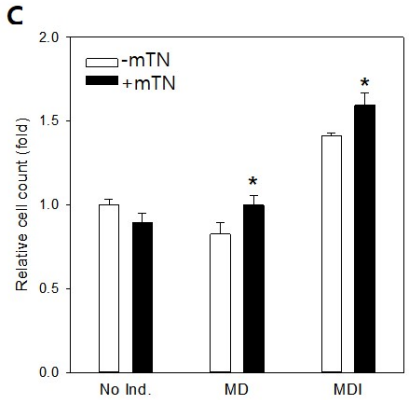

Fig. 3. Enhancement of cell proliferation and MCE by mTN. (A) Proliferation of growing 3T3-L1 cells in TN-del-FBS medium supplemented with $\mathrm{mTN}$ at the indicated concentrations. (B and C) Adipocyte differentiation of growth-arrested 3T3-L1 cells was induced in TN-del-FBS medium with the indicated induction cocktails and $\pm 5 \mu \mathrm{M}$ mTN supplementation. No Ind., no induction. Cell numbers were determined at $24 \mathrm{~h}(\mathrm{~B})$ and $48 \mathrm{~h}(\mathrm{C})$ post-induction using a cell counter. Data $(\mathrm{n} \geq 4)$ with $\mathrm{P}$ values $<0.05(*)$ and $<0.01(* *)$ are indicated.

tion was detected at $24 \mathrm{~h}$, which decreased to a basal level at $48 \mathrm{~h}$. Differentiation induction with MD showed similar changes in the phosphorylation of ERK, the levels of which peaked at $30 \mathrm{~min}$ and $24 \mathrm{~h}$, although the relative phosphorylation levels were different from those obtained by induction with MDI. The effect of mTN on the phosphorylation of ERK was examined at $30 \mathrm{~min}$ and $24 \mathrm{~h}$ post-induction (Fig. 4C-E). mTN supplementation did not alter the expression and phosphorylation of ERK at $30 \mathrm{~min}$ post-induction (Fig. 4C). However, at $24 \mathrm{~h}$ postinduction with $\mathrm{MDI}, \mathrm{mTN}$ significantly increased ERK phosphorylation by 1.8 -fold of the p-ERK/ERK ratio (Fig. $4 \mathrm{D}$ and E). A similar effect of mTN was obtained at $24 \mathrm{~h}$ post-induction with $M D$, showing a 1.7-fold ( $p$-ERK/ERK ratio) increase in the phosphorylation of ERK (Fig. 4D and E). 
A

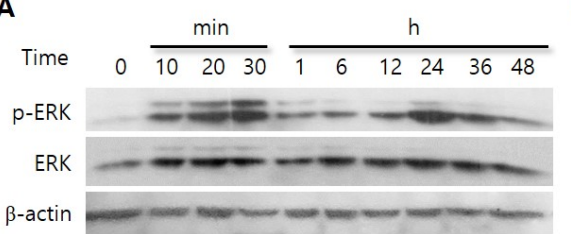

C

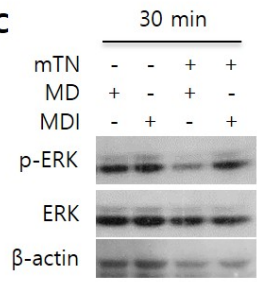

D

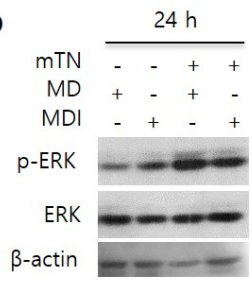

B

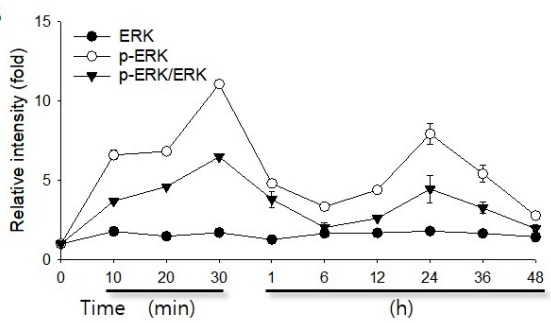

E

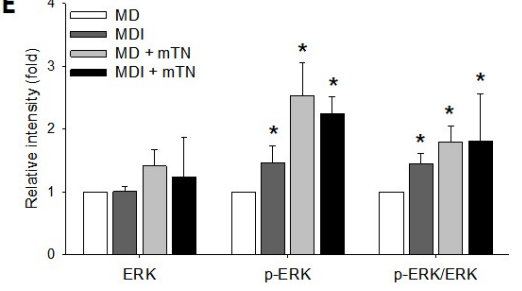

Fig. 4. Increase in the phosphorylation of ERK by mTN. (A) Changes in ERK phosphorylation within $48 \mathrm{~h}$ post-induction with $\mathrm{MDI}$ in TN-del-FBS medium. ERK and phospho-ERK ( $p$-ERK) were analyzed by western blotting at the indicated times. (B) Plot of relative ERK, p-ERK, and $p$-ERK/ERK levels in (A) versus time post-induction. (C and D) Western blot analysis of ERK and p-ERK at $30 \mathrm{~min}$ and $24 \mathrm{~h}$ post-induction with the indicated induction cocktails $\pm 5 \mu \mathrm{M}$ mTN. (E) Plot of relative ERK, $p$-ERK, and $\mathrm{p}$-ERK/ERK levels at $24 \mathrm{~h}$ post-induction. Data $(\mathrm{n} \geq$ 3) with $P$ values $<0.05\left(^{*}\right)$ are indicated.

\section{DISCUSSION}

For adipocyte differentiation, 3T3-L1 preadipocytes were grown to arrest growth in medium containing CS instead of FBS, which contains adipogenic factors that cause spontaneous basal-level differentiation without induction of differentiation. Insulin is an adipogenic serum protein identified by early studies, and it has been included in differentiation induction cocktails $(20,21)$. Previously, we analyzed the bovine serum proteome and identified TN as an adipogenic protein in FBS (18). Another study revealed that mTN requires the conserved kringle-4-binding domain for its adipogenic effect (19). However, there was no evidence that the adipogenic function of mTN is mediated by the activation of Plg involved in remodeling ECM.

In the present study, we demonstrated that the adipogenic function of mTN is mediated by enhancing MCE via ERK signaling, which is a prerequisite for adipocyte differentiation. Since FBS contains $\sim 10-15 \mathrm{mg} / \mathrm{L}$ TN depending on batch-tobatch variations, TN in FBS was removed, and TN-del-FBS medium was used for the adipocyte differentiation of 3T3-L1 cells. Differentiation was markedly reduced by removing $\mathrm{TN}$, and the supplementation of the TN-del-FBS medium with mTN significantly increased the differentiation level. This adipogenic effect was obtained by mTN supplementation at the induction of differentiation, but not after 2 days post-induction, clearly indicating that the protein plays a role in an early phase of adipocyte differentiation (Fig. 1). Moreover, the adipogenic effect of mTN was more significant when inducing differentiation with MDI than it was with MD, and it appeared to be dependent on the insulin dose (Fig. 2A and B). These results suggested that the adipogenic function of mTN could be closely related with the function of insulin in the early phase of adipocyte differentiation.

TN has been suggested as a cancer marker in serum, and is considered to be involved in cancer development and metastasis via the remodeling of ECM and/or the enhancement of cell proliferation (7-9). The effect of TN on cell proliferation is not yet clearly understood, as the results are inconsistent. One study showed that TN enhanced the cell proliferation and progression of colorectal cancer (9), whereas another study indicated that TN inhibited the proliferation of renal carcinoma cells (22). In this study, we found that mTN enhanced the proliferation of growing 3T3-L1 cells (Fig. 2A). In addition, mTN enhanced the proliferation of some cancer cells, but not all tested cells (data not shown). It can be speculated that the effect of mTN (TN) on cell proliferation might be specific to cells with different sensitivities.

More importantly, we found that mTN enhanced MCE, which is the proliferation of growth-arrested 3T3-L1 cells in the early phase of adipocyte differentiation upon induction (Fig. 3C). Moreover, mTN increased ERK phosphorylation, which was consistent with the enhancement of MCE (Fig. 4D and E). Phosphorylation of ERK is a key step in activating MCE via ERK signaling, which has been shown to be enhanced by insulin $(15,23)$. Induction of differentiation in TN-del-FBS medium revealed changes in the phosphorylation of ERK, which rapidly increased to reach the first peak at $30 \mathrm{~min}$ and the second peak at $24 \mathrm{~h}$ (Fig. $4 \mathrm{~A}$ and B). These results are similar to the changes in ERK phosphorylation upon the induction of differentiation in FBS medium (23). However, in TN-del-FBS medium, phosphorylation of ERK at $24 \mathrm{~h}$ was highly significant, while the corresponding phosphorylation in FBS medium was close to a basal level. Interestingly, in the TN-del-FBS medium, insulin significantly enhanced ERK phosphorylation at $24 \mathrm{~h}$ postinduction, but insignificant at $30 \mathrm{~min}$ (Fig. C-E). This delayed effect of insulin on the phosphorylation of ERK could be due to unknown serum factors that were removed in the preparation of TN-del-FBS. mTN increased ERK phosphorylation significantly 
at $24 \mathrm{~h}$, but insignificant at $30 \mathrm{~min}$, which agreed well with the significant enhancement of MCE at $48 \mathrm{~h}$. These results indicate that the enhancement of MCE by MTN is mediated via ERK signaling.

In summary, we discovered that the adipogenic protein mTN plays its role in the early phase of adipocyte differentiation. mTN enhanced MCE that is prerequisite for adipocyte differentiation in the early phase. Consistently, mTN increased the phosphorylation of ERK in the early phase of adipocyte differentiation. Results in this study demonstrate that the adipogenic function of mTN is mediated by enhancing MCE via ERK signaling, although the detailed signaling pathway and regulatory mechanism have yet to be elucidated.

\section{MATERIALS AND METHODS}

\begin{abstract}
Materials
Chemicals were purchased from Sigma Aldrich (St. Louis, MO, USA), unless otherwise indicated. FBS and calf serum (CS) were from Welgene (Gyeongsan, Korea). TN-del-FBS was prepared by partial protein precipitation and fractionation with the addition of polyethylene glycol $8000(10 \%$, w/v) to fetal bovine serum (FBS), as previously described $(24,25)$. Recombinant mTN was prepared in the full-length form fused with $6 \times$ Histag at the $\mathrm{N}$-terminus, as described in the previous report (19). Primary antibodies (anti-ERK $1 / 2$, anti-pErk $1 / 2$, anti-C/EBP- $\beta$, and anti- $\beta$-actin) and secondary antibodies for western blot analysis were from Santa Cruz Biotechnology (Santa Cruz, CA, USA).
\end{abstract}

\section{Cell culture}

Mouse-derived 3T3-L1 preadipocytes were maintained in CS medium (DMEM $10 \%$ calf serum) by incubation at $37^{\circ} \mathrm{C}$ in a humidified atmosphere of $5 \% \mathrm{CO}_{2}$. Adipocyte differentiation was induced as described in (26) with modifications. Preadipocytes 3T3-L1 were grown to $100 \%$ confluency (Fig. 1A D-2) and then incubated for another 2 days to reach growth arrest (Fig. 1A D0). Adipocyte differentiation was induced in FBS medium (DMEN10\% FBS) or TN-del-FBS medium (DMEN10\% TN-del-FBS) by the addition of $0.5 \mathrm{mM} \mathrm{MIX}, 1 \mu \mathrm{M}$ DEX, and 5 $\mu \mathrm{g} / \mathrm{ml}$ of insulin. After inducing differentiation for 2 days, cells were incubated in FBS medium or TN-del-FBS medium supplemented with $5 \mu \mathrm{g} / \mathrm{ml}$ of insulin for another 6 days. Media were changed every 2 or 3 days. Differentiated cells were stained with oil red $\mathrm{O}$, and lipid contents were estimated by measuring the absorbance of isopropanol-extracted dye at $519 \mathrm{~nm}$ (18).

\section{Quantitative real-time PCR}

To determine the expression of adipogenic marker genes, cells were harvested on day 5 post-induction. Total RNA was isolated using TRlzol reagent, and cDNA was synthesized using a cDNA reverse transcription kit (Invitrogen, Carlsbad, CA, USA) according to the manufacturer's protocol. Quantitative PCR amplification was carried out with the following primers: 5'-CCTTTTTCCACCTCCACCTA-3' and 5'-CTCTCGCATCGATCGATTGT- $3^{\prime}$ for C/EBP- $\alpha$, and 5'-CCATTCTGGCCCACCAAC-3' and 5'AATGCG AGTGGTCTTCCATCA-3' for PPAR- $\gamma$, using an ABI 7500 Real-Time PCR system (Applied Biosystems, Foster City, CA, USA) with SYBR green Supermix (Bio-Rad, Hercules, CA, USA).

\section{Determination of cell proliferation and MCE}

Cell proliferation was determined with 3T3-L1 by seeding 1-3 $\times 10^{3}$ cells/well in a 96-well culture plate containing TN-delFBS medium and incubated at $37^{\circ} \mathrm{C}$ in a humidified atmosphere of $5 \% \mathrm{CO}_{2}$ for $24 \mathrm{~h}$. Then, the culture media were supplemented with the indicated concentrations of mTN. After another $24 \mathrm{~h}$ of incubation, the number of viable cells was determined using a D-Plus CCK kit (Donginbiotech, Seoul, South Korea) according to the manufacturer's protocol. For the determination of MCE in TN-del-FBS medium, adipocyte differentiation of post-confluent and growth-arrested 3T3-L1 cells was induced with MIX + DEX (MD) or MIX + DEX + insulin (MDI), with or without $5 \mu \mathrm{M}(100 \mu \mathrm{g} / \mathrm{ml}) \mathrm{mTN}$. Cell numbers were determined at 24 and $48 \mathrm{~h}$ post-induction using a LUNA II automated cell counter (Logos Biosystems, Anyang, South Korea).

\section{Western blot analysis}

Cells were washed twice with ice-cold phosphate-buffered saline and harvested at the indicated incubation times. Harvested cells were lysed in buffer containing $50 \mathrm{mM}$ Tris- $\mathrm{HCl} \mathrm{pH} \mathrm{7.4,}$ $150 \mathrm{mM} \mathrm{NaCl}, 1 \% \mathrm{NP}-40,0.2 \%$ sodium dodecyl sulfate, $1 \times$ protease inhibitor cocktail (Roche Diagnostics, Basel, Switzerland), and $1 \times$ phosphatase inhibitor cocktail (Roche Diagnostics). Soluble proteins were separated by centrifugation at 14,000 rpm and $4{ }^{\circ} \mathrm{C}$ for $30 \mathrm{~min}$, and protein concentrations were determined by bicinchoninic acid assay (Thermo Scientific, Waltham, MA, USA). Proteins were separated by $12 \%$ SDS-PAGE and transferred to a polyvinylidene fluoride membrane, which was blocked in TBS-T buffer $(20 \mathrm{mM}$ Tris pH 7.5, $500 \mathrm{mM}$ $\mathrm{NaCl}, 0.1 \%$ Tween 20) containing 5\% skim milk. The membrane was incubated with the indicated primary antibodies and, after washing, with a horseradish peroxidase-conjugated secondary antibody. Immunoreactive proteins were visualized using a chemiluminescence detection kit, D-Plus ECL Pico (Donginbiotech, Seoul, Korea), according to the manufacturer's instructions. Western blot signals were quantified using Image $X$ software and normalized using $\beta$-actin to determine relative signal intensities.

\section{Statistical analysis}

Data are expressed as means \pm standard errors (SEs). The significance of differences between intracellular lipid contents and gene expression levels was analyzed by an unpaired Student's t-test. A P value $<0.05$ was considered statistically significant.

\section{ACKNOWLEDGEMENTS}

This research was supported by Basic Science Research Program 
through the National Research Foundation of Korea (NRF) funded by the Ministry of Education (2020R1A6A1A03044512) and Yeungnam University research grants in 2020.

\section{CONFLICTS OF INTEREST}

The authors have no conflicting interests.

\section{REFERENCES}

1. Clemmensen I, Petersen LC and Kluft C (1986) Purification and characterization of a novel, oligomeric, plasminogen kringle 4 binding protein from human plasma: tetranectin. Eur J Biochem 156, 327-333

2. Westergaard UB, Andersen $\mathrm{MH}$, Heegaard CW, Fedosov SN and Petersen TE (2003) Tetranectin binds hepatocyte growth factor and tissue-type plasminogen activator. Eur J Biochem 270, 1850-1854

3. Tuxen MK, Soletormos G and Dombernowsky P (1995) Tumor markers in the management of patients with ovarian cancer. Cancer Treat Rev 21, 215-245

4. Hogdall CK, Christensen L and Clemmensen I (1994) [Tetranectin, a plasma and tissue protein-a prognostic marker of breast and ovarian cancer]. Ugeskr Laeger 156, 6190-6195

5. Wewer UM and Albrechtsen R (1992) Tetranectin, a plasminogen kringle 4-binding protein. Cloning and gene expression pattern in human colon cancer. Lab Invest 67 , 253-262

6. Nielsen $\mathrm{H}$, Clemmensen I, Nielsen $\mathrm{HJ}$ and Drivsholm A (1990) Decreased tetranectin in multiple myeloma. Am J Hematol 33, 142-144

7. Christensen L and Clemmensen I (1991) Differences in tetranectin immunoreactivity between benign and malignant breast tissue. Histochemistry 95, 427-433

8. Hogdall CK, Christensen L and Clemmensen I (1993) The prognostic value of tetranectin immunoreactivity and plasma tetranectin in patients with ovarian cancer. Cancer 72, 2415-2422

9. Zhu HF, Zhang XH, Gu CS et al (2019) Cancer-associated fibroblasts promote colorectal cancer progression by secreting CLEC3B. Cancer Biol Ther 20,967-978

10. Wewer UM, Iba K, Durkin ME et al (1998) Tetranectin is a novel marker for myogenesis during embryonic development, muscle regeneration, and muscle cell differentiation in vitro. Dev Biol 200, 247-259

11. Wewer UM, Ibaraki $K$, Schjorring $P$, Durkin ME, Young MF and Albrechtsen R (1994) A potential role for tetranectin in mineralization during osteogenesis. J Cell Biol 127, 1767-1775

12. Park J, Park J, Nahm SS, Choi I and Kim J (2013) Identification of anti-adipogenic proteins in adult bovine serum suppressing 3T3-L1 preadipocyte differentiation. BMB Rep
46, 582-587

13. Armani A, Mammi C, Marzolla V et al (2010) Cellular models for understanding adipogenesis, adipose dysfunction, and obesity. J Cell Biochem 110, 564-572

14. Bost F, Aouadi M, Caron L and Binetruy B (2005) The role of MAPKs in adipocyte differentiation and obesity. Biochimie 87, 51-56

15. Tang QQ, Otto TC and Lane MD (2003) Mitotic clonal expansion: a synchronous process required for adipogenesis. Proc Natl Acad Sci U S A 100, 44-49

16. Sale EM, Atkinson PG and Sale GJ (1995) Requirement of MAP kinase for differentiation of fibroblasts to adipocytes, for insulin activation of p90 S6 kinase and for insulin or serum stimulation of DNA synthesis. EMBO J 14, 674-684

17. Fuhlendorff J, Clemmensen I and Magnusson S (1987) Primary structure of tetranectin, a plasminogen kringle 4 binding plasma protein: homology with asialoglycoprotein receptors and cartilage proteoglycan core protein. Biochemistry 26, 6757-6764

18. Park J, Park J, Jeong J, Cho KH, Choi I and Kim J (2015) Identification of tetranectin as adipogenic serum protein. Biochem Biophys Res Commun 460, 583-588

19. Park J, Ryu DY, Rahman S and Kim J (2019) Adipogenic function of mouse tetranectin and identification of its functional domain. Biochem Biophys Res Commun 519, 645-651

20. Schmidt W, Poll-Jordan G and Loffler G (1990) Adipose conversion of 3T3-L1 cells in a serum-free culture system depends on epidermal growth factor, insulin-like growth factor I, corticosterone, and cyclic AMP. J Biol Chem 265, 15489-15495

21. Kuri-Harcuch W and Green H (1978) Adipose conversion of 3 T3 cells depends on a serum factor. Proc Natl Acad Sci U S A 75, 6107-6109

22. Liu J, Liu Z, Liu Q et al (2018) CLEC3B is downregulated and inhibits proliferation in clear cell renal cell carcinoma. Oncol Rep 40, 2023-2035

23. Prusty D, Park BH, Davis KE and Farmer SR (2002) Activation of MEK/ERK signaling promotes adipogenesis by enhancing peroxisome proliferator-activated receptor gamma (PPARgamma) and C/EBPalpha gene expression during the differentiation of 3T3-L1 preadipocytes. J Biol Chem 277, 46226-46232

24. Park J, Nahm SS, Choi I and Kim J (2013) Identification of anti-adipogenic proteins in adult bovine serum suppressing 3T3-L1 preadipocyte differentiation. BMB Rep 46, 582-587

25. Park J, Jeong J, Cho KH, Choi I and Kim J (2015) Identification of tetranectin as adipogenic serum protein. Biochem Biophys Res Commun 460, 583-588

26. Hossain M, Imran KM, Rahman MS, Yoon D, Marimuthu $V$ and Kim YS (2020) Sinapic acid induces the expression of thermogenic signature genes and lipolysis through activation of PKA/CREB signaling in brown adipocytes. BMB Rep 53, 142-147 